\title{
A Capacitive Sensing Toolkit for Pervasive Activity Detection and Recognition
}

\author{
Raphael Wimmer ${ }^{\dagger}$, Matthias Kranz ${ }^{\dagger}$, Sebastian Boring ${ }^{\dagger}$, Albrecht Schmidt ${ }^{\ddagger}$ \\ ${ }^{\dagger}$ University of Munich ${ }^{\ddagger}$ Fraunhofer IAIS, Sankt Augustin and B-IT, University of Bonn \\ \{raphael,matthias, albrecht\}@ hcilab.org, sebastian.boring@ifi.lmu.de
}

\begin{abstract}
In this paper we present a toolkit for realizing capacitive sensing applications for human-computer interaction in pervasive computing systems. We argue that capacitive sensors - due to their unique properties - are well suited for many pervasive and ubiquitous computing applications and scenarios. We describe the CapToolKit designed to rapidly realize prototypes and systems that are able to detect the presence of humans and objects. Our toolkit also allows the integration of $3 D$ interaction with everyday objects as well as instrumented environments. We illustrate its capabilities by presenting several applications implemented using CapToolKit. The entire system will be open-sourced to allow utilization of our technology within other research projects. By building on the existing toolkit researchers are provided a foundation for developing their own sensor systems, algorithms, and applications.
\end{abstract}

\section{Introduction}

Capacitive Sensing is a technology that allows detection and tracking of conductive as well as non-conductive objects. Its unique features and limitations make it an interesting addition or even alternative to optical and ultrasonic object tracking. While capacitive sensors have found their place in industrial measuring applications, research in capacitive sensing for human-computer interaction has remained scarce. In Section 3 we present the work done on the subject so far and identify the shortcomings of current research. We argue that current research has neither explored all application areas of capacitive sensing, nor does it provide an ample foundation for further research. We especially see the need for a capacitive sensing toolkit which takes the effort of designing sensor circuits from HCI researchers and lets them focus on new application areas for capacitive sensing. In order to be able to conduct research on capacitive sensing ourselves, we had to develop a new toolkit (creatively named CapToolKit) consisting of sensors, controller hardware (CapBoard, Figure 1) and soft- ware tools. CapToolKit is both a testbed for sensor design, and a basis for research on capacitive sensing in pervasive computing. In Section 4 we describe the toolkit, its properties and limitations. While current research focuses on
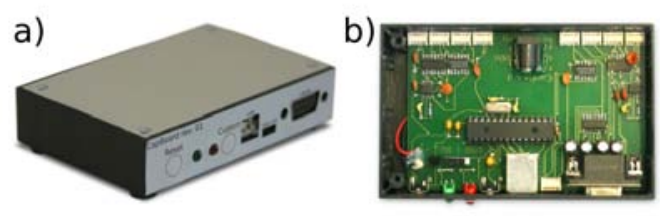

c)

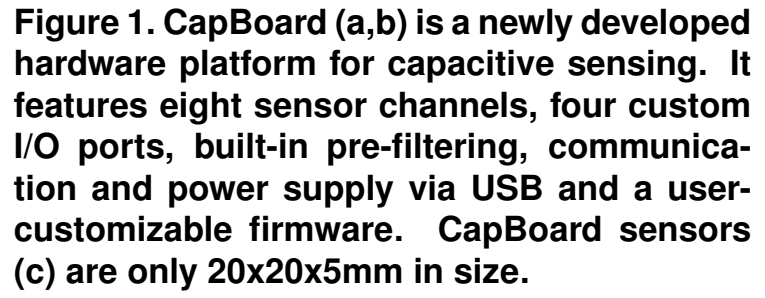

explicit interaction, (e.g. gesture tracking) we see interesting application areas particularly in implicit interaction, namely object detection, activity recognition, and privacyaware instrumented environments. Our vision is that embedding capacitive sensing into everday objects and environments - and providing toolkit support for this - can open a new field for interactive pervasive applications. To prove the benefits of CapToolKit, we have developed and implemented several appliances and scenarios using it. We are aware of limitations inherent to our approach. Equally, we see many interesting areas for further research. In Section 6 we describe a number of improvements to enhance CapToolKit's sensitivity, robustness, and ease-of-use, as well as the future direction of our research.

\section{Capacitive Sensing}

Capacitive sensing is a quite well understood technology. It was employed first in 1919 in a musical instrument called Theremin, where pitch and volume of the sound were 
controlled by the distance between the musicians hands and two antennas. While utilized for industrial measuring applications (e.g. fluid level sensing [2]) and in art projects for many years, the potential of capacitive sensors for human computer interaction is being explored only recently. Exemplarily, several research systems as the School of Fish [7], DiamondTouch [1] or SmartSkin [5] have shown new and interesting applications of capacitive sensing (see Section 3).

\subsection{Basics of Capacitive Sensing}

The simplest capacitor consists of two metal plates put close together without touching each other. When electricity is placed on those plates energy is stored. When electricity is removed and the plates are connected through a circuit, the stored energy initiates a current. Thus, a capacitor works like a small accumulator. The capacity (capacitance) of this simplest capacitor depends on the size of the plates and their distance. The principle holds true for all sophisticated capacitors as well. Using the effect described above, one can infer and track the distance between a sensor and an object from the capacitance they provide. For this, one of the two plates of a capacitor is replaced by the object to be tracked. This object has to provide enough negative charges to counter the positive charge at the sensor plate.

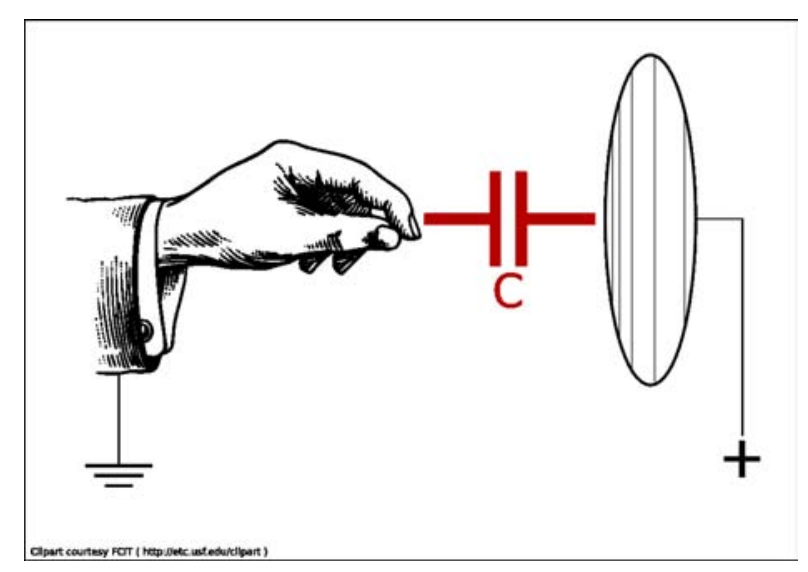

\section{Figure 2. A human hand and a metal plate form a capacitor. From the capacitance of this capacitor the distance between hand and sensor plate can be estimated.}

Objects partially consisting of conductive materials like metal or water are very well suited for tracking. Ideally the object is grounded, as earth provides a quasi endless charge reservoir. However, often the object itself provides a sufficient charge reservoir. Especially the human body does not necessarily need to be grounded in order to act as a good capacitor plate. When the object gets closer to the plate, the capacitance of the so formed capacitor increases. One can measure the capacitance and from this calculate or estimate the distance between sensor plate and object. Capacitive sensors allow measurement of microscopic displacements in the range of micrometers. They are the industry standard for ultra-high precision measurements in many application areas. Furthermore they can also be used for largescale tracking, featuring sensing ranges up to three meters. However, for such large distances to be covered, special measures, like virtual grounds (see Section 4.1), have to be taken.

\subsection{Capacitive Sensing and Electric Field Sensing}

While the principle of capacitive sensing described above is used in the Theremin and most industrial sensors, there are other means to use quasi-static electric fields for tracking conductive objects. Smith [8] coined the term electric field sensing (EFS) for a class of methods measuring electric field strength. He describes three different modes of EFS: Loading Mode, Transmit Mode and Shunt Mode. Loading mode is equivalent to "traditional" capacitive sensing, where field generator and receiver are unified in one electrode. In Transmit Mode the user is coupled to an antenna and prolongs its electric field. Thus the capacitance between his hand and a receiving sensor plate can be measured. However, this mode requires the user to be connected to an electrode. Shunt mode utilizes the effect that a conducting object near an emitter-receiver electrode combination shunts part of their electric field to ground, acting as a shield. Thus the electric field measured at the receiving electrode decreases as the object approaches. Shunt mode offers the advantage of getting $\frac{n *(n-1)}{2}$ measurements from $n$ sensor plates. For details on EFS see [7]. A discussion why Loading Mode was choosen for CapBoard can be found in Section 4.4.

\subsection{Limitations of Capacitive Sensing}

While capacitive sensing offers almost 'magical' features there are some limitations that have to be taken into account when employing this technology.

- Ambiguous Data. Capacitive sensors offer only onedimensional data: the capacitance provided by the electrode. Thus the system has to decide, what a capacitance change means. Most times it is caused by a conductive object moving farther from or closer to the sensor electrode. However, changes in the dielectric value of the isolator between object and electrode, which is usually air, also affect the capacitance. Another possible reason may be a change in size of the object. This is e.g. the case, when a hand is clenched 
to a fist. Sometimes two of the three parameters can be controlled, leaving only one cause for capacitance changes. For example, when tracking a human hand, the dielectric value of the air between hand and sensor is likely to stay constant. The size of the hand, while changing with orientation, stays in the same order of magnitude. However, resulting from this ambiguity of sensor data, a capacitive sensor can not distinguish between a small object at a short distance and a big object or two small objects at a greater distance. Both effect the same capacitance to the sensor electrode. This means that lots of additional information has to be known to correctly interpret sensor data. However, adding supplementary sensors to a setup reduces ambiguity. By placing an additional capacitive sensor opposite to the first one, the position of one object in between them can be calculated regardless of its size as the distances measured by the two sensors have to add up to the distance between the sensors. Applying different sensor technologies can enhance the quality of acquired data, too. An additional, inherent limitation is that capacitive sensors cannot detect differences in texture, weight or shape of objects. Thus different hands cannot be distinguished. For simultaneous multi- user applications, other means of identifying users have to be found. For example, DiamondTouch infers who is touching where by time-slicing the electric field and detecting which user is exposed to it at a certain time.

- Quickly Decaying Resolution. A major hurdle for incorporating capacitive sensors in pervasive applications is their limited range. If the size of the object to be tracked and the dielectric value of the isolator can be controlled, the capacitance measured by a sensor depends mainly on the distance between electrode and object. The generic equation for the capacitance of a capacitor, $C \approx \frac{\epsilon A}{d}$, implies that it is inversely proportional to the distance between the plates. However, this is not the case for greater distances between the plates. The farther apart the two plates are, the smaller their overlapping area gets relatively to their surrounding Thus a more realistic model is $C \approx \frac{\epsilon A}{d^{x}}$; where $x$ is between $1-3$, depending on the environment. This rapid decrease of capacitance with increasing distance poses a problem when trying to track objects at distances greater than $\approx 10 \mathrm{~cm}$. The lower resolution has to be accounted for, e.g. by placing another sensor opposite of the first so that the object is always close to one sensor. Furthermore, additional sensor data and sophisticated filtering can be useful.

- Exposure to External Influences. As a result of the ambiguous data mentioned above, capacitive sensor measurements are also exposed to other, unwanted influ- ences. If conductive objects which shall not be tracked get into sensor range, they increase capacitance, tainting the measurements. objects. This includes people walking by a sensor, additional hands, or coffee cups being put near a sensor. Other unwanted influences include electric fields emitted by electric devices or humidity and temperature changes. Electric fields also negatively affect measurements. Changes in the dielectric (like increasing humidity) may cause erroneous measurements. Additionally, capacitors are sensitive to temperature changes. As many different effects influence measurements, capacitive sensors usually have to be calibrated, relating measured capacitance to proximity. Depending on the needed sensitivity, these calibrations have to be done regularly, especially when the environment of the sensor changes.

\subsection{Advantages of Capacitive Sensing}

When keeping in mind the aforementioned limitations, capacitive sensors can be employed in a variety of settings. It features completely different capabilities and limitations than for example optical tracking. Some of the properties are especially useful in instrumented environments, where sensors should be unobtrusive as well as reliable. In other application areas capacitive sensors may supplement different sensing technologies. Some of the advantages of capacitive sensing are:

- No line-of-sight needed. While non-conductive objects like glass, plastics or wood change the measured capacitance through their dielectric value, this influence is low. When the distance between sensor electrode and non-conductive object stays the same, no capacitance changes are effected. In addition, these objects usually do not decrease sensor resolution, but even increase it. This is due to their dielectric constant, which is higher than that of air, causing the capacitance to increase slightly. This property enables sensors to be installed in walls or inside casings. However, the thicker the material, the greater the minimal distance between sensor electrode and tracked object - and thus the lower the available maximum resolution.

- Efficient data acquisition. Post processing and analysis can be done in a microcontroller. By lowering the update rate, resolution can be easily increased.

- Cheap hardware. Capacitive sensors can be built with few inexpensive off-the-shelf components. CapBoard, the capacitive sensing hardware described in Section 4 supports eight sensors and has material costs of less than US\$ 50 for prototypes. The material costs for a single sensor are less than US\$ 1 , even in small quantities. 
- High speed. The update rate of a capacitive sensor is usually up to $100 \mathrm{~Hz}$. However, Even faster data acquisition is possible, resulting in a worse signal-to-noise ratio. Even with four-sample averaging the resulting acquisition time of $40 \mathrm{~ms}$ is well below the threshold of $100 \mathrm{~ms}$ deemed as acceptable latency for humancomputer interfaces [6]. Due to the straightforward data they provide, postprocessing is usually limited to fast band-pass or averaging filters, adding minimal latency.

- Small size sensor. Sensor electrodes at a size of $2 \mathrm{~cm}$ in diameter already offer a sensor range of more than $10 \mathrm{~cm}$ with resolutions in the millimeter range. Even smaller electrodes, etched into the circuit board, can be used for ranges of up to a few centimeters. Larger electrodes increase sensor range. The sensor circuit can be built as small as one square centimeter with standard parts or even smaller using custom ICs.

\section{Related Work}

The technology of capacitive sensing itself is already part of today's computers, e.g. in the touchpads of current laptops. There, input is limited to a very small range of sensor to hand, typically less then $1 \mathrm{~cm}$. We extend the sensing range to explore the impacts on the way input to a system can be generated. However, using capacitive sensing for medium-scale position tracking has not been pursued so far.

Smith et Al. [8] and Zimmerman et Al.[10] explored the potentials of electric field sensing as input modality. Especially Joshua R. Smith implemented many systems using shunt mode electric field sensing. Some systems allow gesture tracking in two dimensions. A mobile phone with an integrated EFS module measured the distance between phone and head. This was used to determine radiation exposure of the user's brain. This research on electric field sensing, unfortunately, was discontinued after Joshua R. Smith left the MIT Media lab.

Jacky Lee et al. [4] developed a 3D interface device for $\mathrm{CAD}$ workstations which uses capacitive sensing. This device (iSphere) only measures three different states (distant, close, pressure). The user needs to touch the iSphere for interaction. Interaction at a distance is not supported.

The DiamondTouch table proposed by Dietz et al. [1] uses a grid of metal strips below a tabletop to sense finger positions. A digital projector overlays a computer screen onto the table. The strips of the grid emit - time-multiplexed - an electric field. This design is practically a huge touchpad, very similar in principle to those used in notebooks or the Apple iPod. While touchpads implement loading mode electric field sensing, DiamondTouch utilizes the transmit mode. Two features make DiamondTouch special: Its ability to track more than one finger (which is also possible with recent touchpads), and its ability to discriminate different user's hands. This is made possible by seating all users on metal chairs which are capacitively coupled to the user sitting in them. The user in turn is capacitively coupled to the DiamondTouch grid. By determining which grid strips are active when an electric field is sensed in the chair the system can detect where each user is touching the display. Thus DiamondTouch allows intuitive collaborative interaction on a computer screen. The emitted field of the DiamondTouch table though is only capable of detecting direct touch events. The user has to have direct contact to the table.

SmartSkin by Jun Rekimoto [5] also uses capacitive measurement for user-surface interaction. Again, a fine array of emitters is placed below the surface for achieving the sufficient resolution which is needed for intuitive human-surface interaction. The measuring range in the $\mathrm{Z}$ axis is about 5 $\mathrm{cm}$. SmartSkin is only capable of detecting touch or tagged objects. Untagged objects as they are common and natural are hard to use with the SmartSkin.

EtherTouch [3] is a commercial electric field sensing IC for mobile devices like PDAs or mobile phones. It is connected to $\mathrm{X}, \mathrm{Y}$, and $\mathrm{Z}$ electrodes all together on a thin plastic board. As Ethertouch implements transmit mode electric field sensing, the user has to be capacitively coupled to the emitter. With handheld devices this is very easy to accomplish. The manufacturer claims to reach a sensitivity of $4 \frac{a F}{\sqrt{H z}}$. As no actual devices with this chip are available the usefulness of the IC for pervasive computing applications can not be estimated. Our measurements suggest that CapBoard sensitivity is about in the same magnitude as the EtherTouch. In close ranges up to $10 \mathrm{~cm}$ either solution should provide sufficient resolution.

Thracker [9] has been our first try on capacitive sensing hardware. Four resonating circuits implemented with NAND gates drive counter ICs. Their value is read out at fixed intervals by a USB interface IC (IO-Warrior 40). Thracker's resolution was limited to 8 bit per channel. Its usable range was about $20 \mathrm{~cm}$. This proved to be adequate for tracking a hand in front of a 12.1" display with at least $2 \mathrm{~cm}$ resolution. However, Thracker could not be easily calibrated for changing environments. Due to its hardware design, the signal-to-noise ratio was unsatisfactory. Board schematics and assembly instructions for Thracker are available online ${ }^{1}$.

While the mentioned systems show highly interesting applications for capacitive sensing, current research still lacks both depth and broadth. We feel that there are still some questions unanswered and a lot of hurdles to be overcome for researchers interested in utilizing capacitive sensing.

\footnotetext{
${ }^{1}$ http://www.hcilab.org/projects/gestureui
} 
In our view major shortcomings of current research are:

- Limited application areas. Most systems known to us focus on explicit interaction with the device. While some art installations use capacitive sensing to trigger events if someone approaches, capacitive sensing for implicit interaction - as desired in ubicomp and pervasive scenarios - has not been researched in depth.

- Difficult to reproduce. Despite capacitive sensing being basically a very primitive technology, many researchers have seen the need to design complex specialized hardware in order to overcome some of the limitations of capacitive sensing. From studying the related work it seems that many researchers have difficulty implementing capacitive sensing hardware with sufficient resolution for useful applications.

- No real data available A lack of data on sensor performance discourages new designs and makes it difficult to determine the quality of a capacitive sensing solution. Most publications and technical datasheets omit hands-on figures regarding sensor range, resolution and reliability. Often no actual evaluation of the proposed system has been conducted. Regularly research papers describe actions which can be performed using the proposed system without mentioning its resolution and error rate. Discussing a system's problems and limitations often is not regarded necessary, either.

The lack of reliable data, together with the fact that only renowned researchers seem to be able to build working systems, appear to us as the main shortcomings of current research in capacitive sensing.

\section{The CapToolkit}

We feel that capacitive sensors have a high potential for human-computer interaction in pervasive computing systems. However, due to the aforementioned problems concerning research and implementation of such systems, we see the need for ready-to-use hardware and software to quickly evaluate and integrate capacitive sensing into new prototypes. As none of that existed up to then, we built our own capacitive sensing toolkit, consisting of flexible sensor hardware, microcontroller firmware and software tools. CapToolkit was designed to support sensing interaction in $3 \mathrm{D}$, recognizing activities, and detecting and discriminating objects. It was not our goal to develop hardware that excels in amount of features or sensor range. Our main goals with the toolkit are to:

- provide cheap, easy to build and to use hardware

- provide open-source, cross-platform tools to analyze and visualize the data
- offer hands-on tips and guidelines for implementing custom systems

- enable researchers to improve and extend the system to fit their needs

- make it easy for non-engineers to build new sensing applications

- offer reliable data on range, resolution and error of the hardware, allowing other systems to be compared to this hardware

We will now describe the toolkit components and some example applications we so far built with our system.

\subsection{Toolkit Hardware - CapBoard}

In this section, we present in detail our capacitive sensing hardware, the so-called CapBoard (see Figure 3). It aims to provide a cheap, flexible, and open-source hardware design, device firmware and host software for capacitive sensing interfaces.
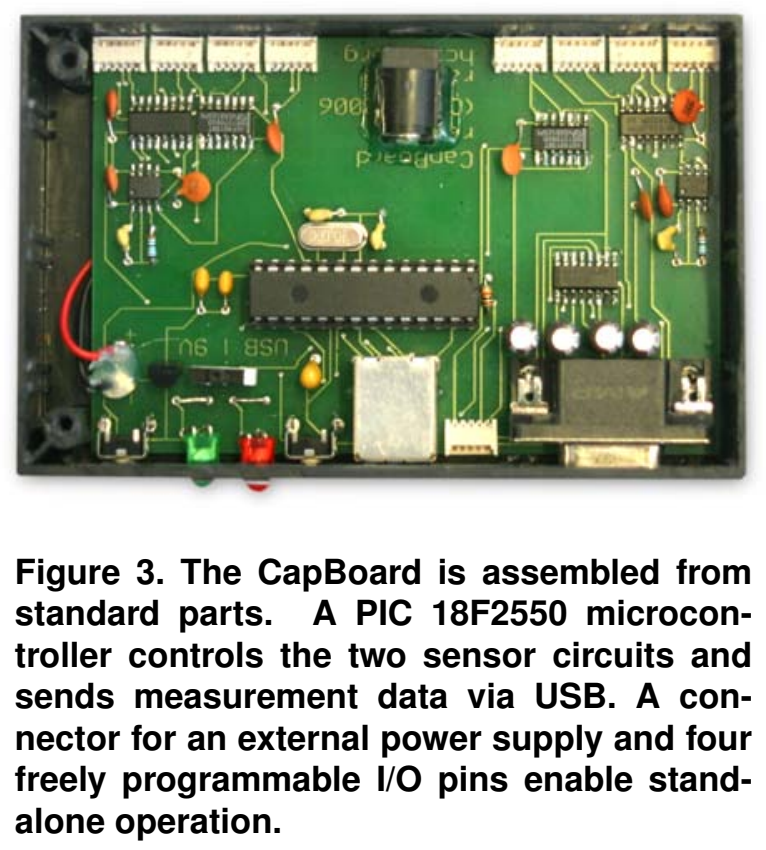

Each CapBoard allows up to 8 capacitive sensors to be read out at an update rate of 25 to $100 \mathrm{~Hz}$. The data is acquired by a PIC microcontroller and published over USB, which also provides power to the system. This reduces the necessity for additional power supply and therefore facilitates easy installation. Complementary sensors can be connected to four analog/digital I/O ports. Acquisition time, filtering, and output format can be easily adjusted. A bootloader firmware allows upload of custom firmware via USB, 
and thus makes fast iteration cycles possible. USB communication is currently using a custom protocol, but support for e.g. the Human Interface Device (HID) Class is planned.

The sensor part is based on the ThereminVision-II hardware $^{2}$ by Terry Fritz. A sensor consists of a sensor board and a sensor electrode. Core of each sensor board is a LMC555 timer IC. Depending on the capacitance of a connected capacitor the LMC555 outputs a non-linear frequency up to $3 \mathrm{MHz}$. A rapidly changing, quasielectrostatic field is generated around the electrode.

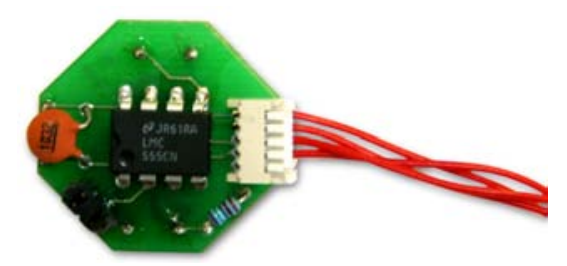

Figure 4. A sensor board, measuring only $20 \times 20 \mathrm{~mm}$. It is connected to the CapBoard with a 4-wire cable. Different sensor electrodes can be attached to accommodate space limitations and range requirements.

The capacitance provided by a small sensor electrode of $10 \mathrm{x} 10 \mathrm{~cm}$ is between $\approx 30 \mathrm{pF}$ (empty room) and $\approx 46 \mathrm{pF}$ (hand in a few millimeters distance). The frequency of the LMC555 is set to about $3 \mathrm{MHz}$ and changes to about $2 \mathrm{MHz}$ when a hand is placed almost on top of the plate. Capacitance and frequency vary depending on antenna size and shape, circuit design, sensor placement and environmental conditions. An additional capacitor can be added in parallel to decrease the frequency.

As two electrostatic fields taint each other, two sensors should not be active near each other at the same time. Thus, each sensor can be enabled and disabled via a digital signal. As our sensor boards consist only of one IC and some resistors and capacitors, they are very cheap (less than US\$ 1 per sensor) and small-size. Current sensor boards are about $20 x 20 \mathrm{~mm}$. Using only small SMD (surface mounted device) parts a sensor size of $10 x 10 \mathrm{~mm}$ is easily possible. The sensor board is depicted in Figure 4.

To each of the 8 sensor channels provided by CapBoard, one sensor can be connected. A four-wire-connector provides power to the sensor, controls its state and is used for data transmission. The sensor puts a square-wave signal with a variable frequency onto one wire. In the CapBoard the frequency of a reference timer $(2 \mathrm{MHz})$ is subtracted from the measured frequency. This is done by a D-type flip-flop. Thus small absolute frequency changes result in rather large relative changes. In four measurement passes

\footnotetext{
${ }^{2}$ http://www.thereminvision.com
}

two sensors are activated and read out at a time. This reduces overall acquisition time but requires the concurrently active sensors to be placed some distance apart. Section 4.5 offers recommendations for sensor spacing.

When measuring the frequency of a signal the resolution depends linearly on the acquisition time. If the signal is read for $10 \mathrm{~ms}$ the smallest frequency change that can be detected is $100 \mathrm{~Hz}$. When measuring a channel for $100 \mathrm{~ms}$ frequency changes of $10 \mathrm{~Hz}$ can be detected. Thus there is always a tradeoff between resolution and update rate when measuring frequencies. The update rate of CapBoard can therefore be adjusted from 25 to $100 \mathrm{~Hz}$, depending on the factors mentioned above.

The communication protocol between CapBoard and host computer is kept deliberately simple and straightforward, following the KISS principle: keep it simple, stupid. Commands sent to CapBoard consist of a one-byte command ID and zero or more parameters. The command IDs are usually menmonics that are easy to rememeber. For example 'A' and ' $Z$ ' start and stop measuring, 'E2' enables sensor channel 2, and 'D2' disables channel 2. 'L21' lights the second LED on CapBoard, will 'L20' is used to turn it off. Acquisition time is set using 'Titime in milliseconds ¿'. Upon each command CapBoard sends a reply indicating success or failure. Measuring data can be delivered in various formats, depending on the needs of the connected application. Everything can be configured and altered during run-time of the system.

\subsection{Toolkit Software}

CapToolkit comprises software for accessing CapBoard via USB and additional tools to visualize the sensor data. We provide two interfaces for accessing the CapBoard data. The first one streams sensor data over TCP or UDP sockets. This allows integration of CapBoard in every system that can send and receive data over sockets. While this bridging software needs to be run on the computer the CapBoard is connected to, applications that process the data can be run on any networked computer. CapBoard uses a plain-text protocol to send data and receive commands. Starting the bridging software and connecting to the TCP socket with HyperTerminal or netcat is everything needed to view sensor data. Thus CapBoard makes rapid development of prototypes and troubleshooting easy.

The second interface uses the sensor/actuator middleware Player/Stage and publishes the sensor data via the analog i/o interface (AIO). This open source middleware is widely used in the robotics domain and well supported. The data can be accessed with a great variety of programming languages as well. By providing these two interfaces we hope that every researcher interested in using the system can easily access the data and use it for his research. Both 
tools allow the capture and logging of the desired sensor values and to use it in any other application, e.g. for activity recognition.

We also developed several tools for visualizing the sensor data. CapBoard Analyzer (Figure 5) is a Java-based GUI that displays the values of the desired sensors in real-time. It connects to a TCP socket and plots sensor values over time. The tool also allows to control several parameters of the CapBoard firmware such as acquisition time, channels enabled or filtering.

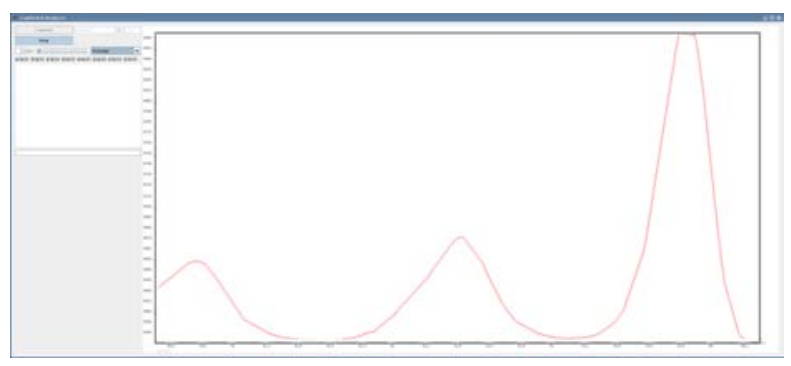

Figure 5. CapBoard Analyzer displays capacitance values for each sensor in real-time and plots it over time. A GUI enables the user to quickly enable and disable sensors, select filters, or adjust the acquisition time. The data can be logged to a file for further review.

\subsection{Integrating CapBoard into existing hardware}

The sensor electrodes can be arbitrarily shaped to accommodate for size constraints. However, electrode size and shape define the sensor's range and sensitivity: the larger the electrode the greater the sensing range. A metal plate electrode provides high sensitivity (and range) in the orthogonal axis. Objects in the other two axes have only very limited influence on the sensor's capacitance.

On the other hand a spherical electrode can be used for omnidirectional sensing, e.g. to provide simple proximity detection. Spherical electrodes also simplify data extraction from measurement data as they can be described as single points instead of planes. However, it is often difficult to integrate a sphere into hardware designs. In scenarios like gesture tracking spherical electrodes may introduce more noise than simple bi-directional plates, as the ratio of sensor area facing the object to total sensor area is much lower.

\subsection{Loading Mode and Frequency Measuring}

Most existing EFS systems implement transmit mode (DiamondTouch, SmartSkin) or shunt mode (School of Fish). Those are expected to provide better resolution than the loading mode EFS implemented in CapBoard. For our Toolkit we have chosen loading mode for three reasons:

- placement of sensors and data analysis is less complex than in shunt mode

- for most pervasive computing applications it is not feasible to connect the user to be tracked to an electrode like it is necessary with transmit mode EFS.

- loading mode EFS affords the simplest implementation, thus offering less points of failure, and minimizing calibration.

Most commercial sensors output their measurements as a voltage level instead of signal frequency as CapBoard sensors do. Using signal frequency has advantages and disadvantages.

High-frequency signals are more prone to noise than voltage levels. However, our measurements do not indicate any influence of external signals on the measured frequency. Another drawback of our method is that precisely measuring a frequency takes more time than measuring voltage levels. We argue that for most pervasive computing applications an update rate of $25 \mathrm{~Hz}-100 \mathrm{~Hz}$ is quite sufficient.

Measuring a frequency avoids two D-A (sensor) and A$\mathrm{D}$ (microcontroller) conversions. Thus no conversion errors can occur. Additionally, signal frequency stays the same even when transmitted over long cabling. Voltage levels drop, however, with distance. Therefore we believe that CapBoard provides ample resolution and range for most applications.

\subsection{Range, Resolution, Reliability}

A major concern in developing the sensor toolkit was good documentation of its features and limitations. The information we missed most in current research were "How far does the sensor reach?", "What resolution does it provide?", and "What are its limitations?". We will try to answer these questions for CapToolKit. While it is not possible to describe all our measurements and findings in this paper we present a thorough overview of them this section. Detailed data can be accessed at our website.

Measuring Range greatly depends on the size of the sensor electrode. As a rule of thumb, the usable sensor range is about two or three times the diameter of the electrode. Actual maximum range depends on acquisition time, environment, and object to be tracked. In our tests we could detect a human body at a distance of $\approx 100 \mathrm{~cm}$ and track hand movements at a distance of $\approx 50 \mathrm{~cm}$ using a $10 \times 10 \mathrm{~cm}$ sensor electrode.

Spatial Resolution greatly depends on the distance between object and sensor plate. CapBoard is able to detect capacitance changes of $100 \mathrm{aF}$ at an update rate of $50 \mathrm{~Hz}$. 
Using a $10 \times 10 \mathrm{~cm}$ electrode this offers a spatial tracking resolution of $10 \mathrm{~mm}$ at a distance of $25 \mathrm{~cm}$. Figure 6 shows actual spatial resolution for different electrode sizes and distances.

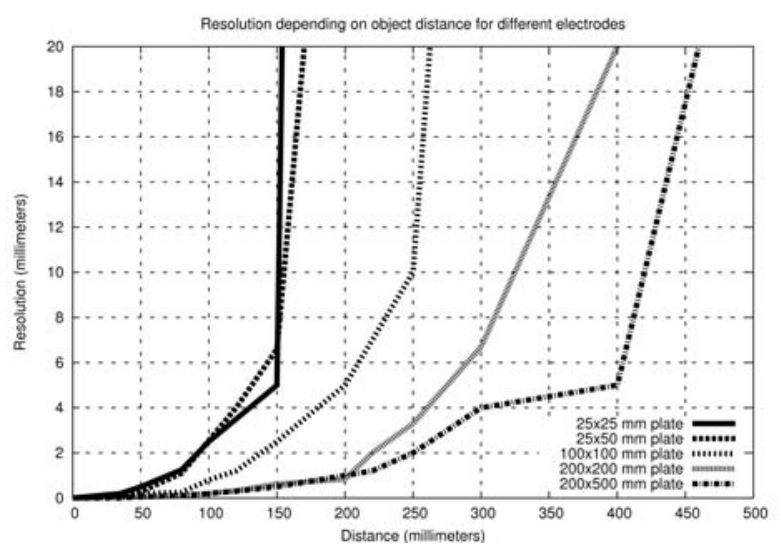

Figure 6. Comparison of CapBoard sensitivity for different sensor plates. For all measurements, the setup described in Section 4.6 was employed.

Reliability and Robustness of CapBoard are sufficient for long-term, heavy-duty data acquisition. Due to its low complexity, open source firmware and modular design the hardware will work reliably and unattended. However, environmental factors like temperature or humidity have a measurable influence on capacitive sensors. Thus the system should be re-calibrated if one of these factors changes. We are considering an auto-calibration feature and/or temperature compensation for the next version of the toolkit. A major problem with capacitive sensors is their susceptibility to other electric fields. Thus, care should be taken to keep those fields away from the sensors. When employing concurrently active sensors, they should be seperated by an ample distance. Figure 7 shows the connection between sensor size and necessary spacing.

Another related problem is electrically separating sensor circuits. In our current hardware revision the sensors taint each other slightly but recognizably. By incorporating virtual grounds into the next revision, we hope to improve CapBoard's signal-to-noise ratio.

\subsection{Comparison to existing solutions}

As has been described in Section 3 there is almost no data on the capabilities of existing capacitive sensors. The only data on EFS resolution we found in the related work, was a small figure in the first paper on EFS [10]. While no additional information was given e.g. on maximum range

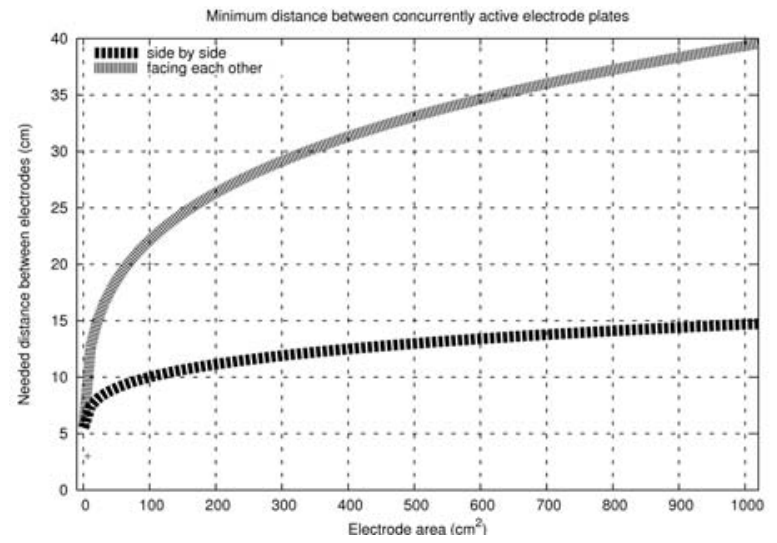

Figure 7. To avoid two concurrently active electrodes tainting each other's electric field, a certain distance between them should be kept. Often smaller distances are possible if a worse signal-to-noise ratio is acceptable.

of their solution, the measuring setup was described in detail. We replicated this setup as exactly as possible. The original setup consisted of a transmitting and a receiving metal plate, each $2.5 \mathrm{~cm} \times 2.5 \mathrm{~cm}$ in size, spaced $15.2 \mathrm{~cm}$ on center. An aluminium tube acted as a surrogate arm. The spatial resolution at certain distances between sensor plates and surrogate arm was measured. Due to the different sensing modes employed, we replicated the tests with a single $2.5 \mathrm{~cm} \times 2.5 \mathrm{~cm}$ and subsequentially a single $2.5 \mathrm{~cm} \times 5 \mathrm{~cm}$ metal plate instead of the two plates used by Zimmerman et al. Depending on the actual implementation of such a system, one of the two measurements provides a realistic prediction of CapBoard's sensitivity. Additionally, we were not able to acquire a $48.3 \mathrm{~cm} \times 7.6 \mathrm{~cm}$ aluminium tube and used one with a size of $48 \mathrm{~cm} \times 8 \mathrm{~cm}$. To verify the aptitude of the aluminium tube as a surrogate arm, we repeated the measurements with a real arm, affixed to a wooden frame to reduce trembling. The real arm offered slightly better tracking performance than the surrogate arm.

As can be seen in Figure 8 CapBoard offers a significantly lower spatial resolution than the Classic Fish, once distance exceeds $20 \mathrm{~cm}$. We believe that this is caused by the different sensing modes (loading mode vs. shunt mode EFS) employed. We also believe that CapBoard's measuring range can be enhanced significantly in future redesigns. However, CapBoard will probably never reach the ranges possible with shunt mode EFS. As has been explained in Section 4.4, this was deemed acceptable for many applications. 


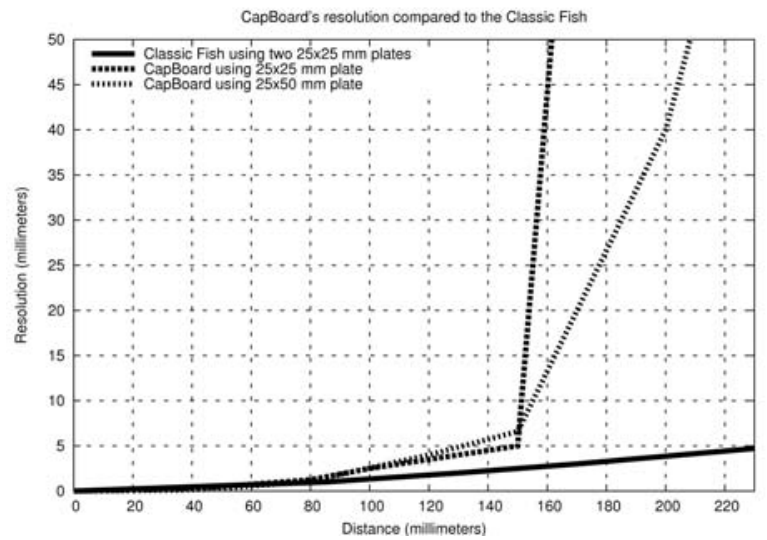

Figure 8. Due to the different working principles, CapBoard offers significantly lower range and resolution than the Classic Fish presented by Zimmerman et al. At distances up to $15 \mathrm{~cm}$ both systems show similar properties, however.

\section{Toolkit Examples}

In this section, we present several example applications we realized with the toolkit presented above. These short usage scenarios are intended to demonstrate that CapToolKit is a suitable basis for prototyping, and show possible new application areas for capacitive sensing.

CapKeyboard. An earlier version of our hardware has been attached to two small metal plates which are affixed to a computer keyboard. Moving her hand above the plates the user can intuitively scroll in large documents, avoiding repetitive and stressing use of the mouse scroll wheel. Absolute positioning in a large document can be done but has shown less intuitive than relative positioning. Another application we implemented (and use) is 'display switching'. Many computers have two displays attached to them. Switching between two windows on different displays either requires furious 'Alt-Tab'ing or repositioning the mouse. With CapKeyboard and 'focus follows mouse' a simple wipe over the sensors moves the mouse pointer on either display, activating the window on it. Thus switching between keyboard and mouse can be reduced. This feature - while not really impressive - has shown it's usefulness in everyday work.

CapTable. CapTable is a standard wooden IKEA table enhanced with capacitive sensors. Eight $20 \times 20 \mathrm{~cm}$ sensor electrodes arranged in a slightly modified $4 \times 2$ layout enable CapTable to sense activity and discriminate objects. Initial tests showed that our setup can track people moving in the proximity of the table and hands hovering above the table. Tracking works at distances of up to $70 \mathrm{~cm}$ above the table, and about the same distance around the table. CapTable is also able to distinguish different objects on top. The objects do not have to be incorporate conductive material, as long as they have a different dielectric constant than air. We could e.g. reliably tell apart three almost identical empty coffee mugs by their dielectric properties. For preliminary visualisation of activity data, we have written CapTable Visualizer (Figure 9). It allows to display live video or a captured video stream overlayed with sensor data from CapTable. The purpose of this tool is to combine sensor data and video as starting point for activity recognition. It displays all relevant data concurrently in a convenient, humanunderstandable format.

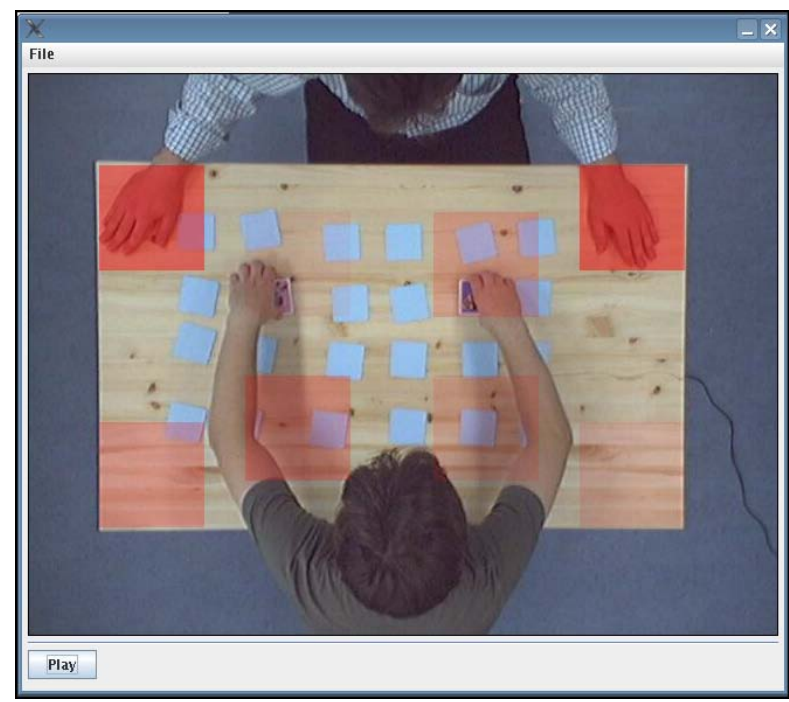

Figure 9. CapTable Visualizer displays a video of the scene with capacitive sensing data overlayed. In this screenshot the unprocessed sensor values are shown as saturation of squares.

CapShelf. CapShelf is a regular kitchen shelf with sensor plates connected to the CapBoard hardware. The kitchen shelf has two compartments. We can detect and track a human hand reaching for objects placed in the shelf, e.g. cleaning utilities. This allows us to detect which object has been taken or put back in the shelf, much like an RFID reader system would allow. Additionally, we can derive the position and duration of this activity to provide a richer set of data for this type of context.

Fluid Level Sensing. Capacitive sensors are widely used for measuring fluid levels. CapBoard can be used for this task, too. In our setup we placed a Bavarian beer bottle next to a $10 \times 10 \mathrm{~cm}$ electrode. Thanks to the small distance between sensor and bottle we were able to reliably and precisely measure the fluid level in the bottle (see Figure 10). 

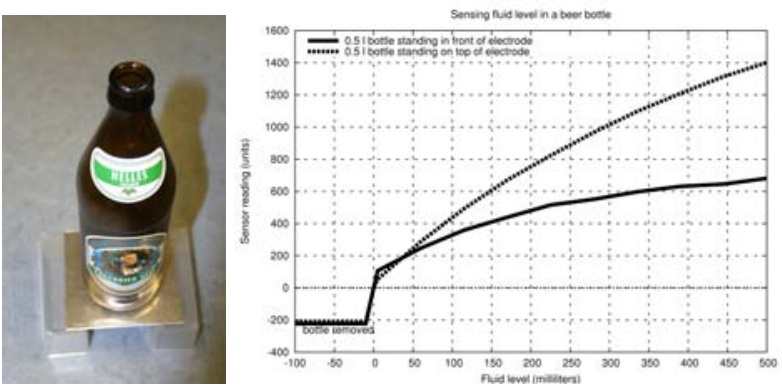

Figure 10. CapBoard also allows tracking of fluid levels. The amount of beer remaining in a bottle can be precisely determined. No smoothing has been applied to the sensor data.

We see application areas for such sensors e.g. in fridges or kitchen cabinets that monitor the amount of beverages remaining and ring an alarm if supplies are nearly used up. By calibrating the firmware for a certain bottle type, the number of beer bottles in the fridge can be determined. Thus unhealthy consumption habits may be recognized early and appropriate measures be taken.

\section{Discussion and Future Work}

We introduced the CapToolkit as out-of-the-box tool for prototyping human-computer interaction using capacitive sensing technology. We described the hard- and software components of the system and prototypically developed several applications using the CapToolkit.

The hardware is easy to install and very cheap to build, even in low numbers. Everyday objects can easily be augmented with the sensing system introduced. The data that can be derived is useful for a wide range of applications, but still some advanced features are missing. This will be part of the future work as discussed below. The sample applications we built help the researcher to get an initial understanding of the data and can help to interpet the data for a full qualified context. The toolkit itself is a powerful prototyping tool for quickly developing context-aware applications.

As we believe that there is an enormous potential for human-machine interaction and context-awareness using capacitive sensing, we will provide detailed guidelines and configuration information. This will allow researchers as well as non-computer scientists to include capacitive sensing systems in their appliances. We will try to cover some of the most common arrangements of sensors e.g. for tables, shelves and other furniture, giving information on how many plates in what position will be needed to reliably de- rive a certain activity or context. This will also comprise a visualization toolkit of which we already showed an early version in this papers.

We thereby hope that CapToolKit helps researchers utilize the possibilites capacitive sensing offers, much like e.g. the AR Toolkit did for augmented reality applications. We expect that this will have an impact on the role of capacitive sensing in pervasive computing systems. Schematics, assembly instructions, source code and documentation can be found at http: //www. capsense.org.

\section{Acknowledgements}

The work has been conducted in the context of the research projects Embedded Interaction ('Eingebettete Interaktion') and Fluidum, and was funded by the DFG ('Deutsche Forschungsgemeinschaft'). We would like to thank the people at the Intelligent Autonomous Systems Group at the Technical University of Munich for providing a great environment for our CapTable.

\section{References}

[1] P. Dietz and D. Leigh. Diamondtouch: a multi-user touch technology. In UIST '01: Proceedings of the 14th annual ACM symposium on User interface software and technology, pages 219-226, New York, NY, USA, 2001. ACM Press.

[2] P. Dietz, D. Leigh, and W. Yerazunis. Wireless liquid level sensing for restaurant applications, 2002.

[3] Ethertouch Inc. Ethertouch. http://www. ethertouch. com/, visited January 2006.

[4] C.-H. Lee and T. S. Yuchang Hu. iSphere: a Proximitybased 3D Input Interface. CAAD Futures, 2005.

[5] J. Rekimoto. Smartskin: an infrastructure for freehand manipulation on interactive surfaces. In $\mathrm{CHI}$ '02: Proceedings of the SIGCHI conference on Human factors in computing systems, pages 113-120. ACM Press, 2002.

[6] B. Shneiderman. Designing the User Interface: Strategies for Effective Human-Computer Interaction. Addison- Wesley, 3rd edition, 1997.

[7] J. Smith. Electric Field Imaging. PhD thesis, MASSACHUSETTS INSTITUTE OF TECHNOLOGY, Feb. 1999.

[8] J. Smith, T. White, C. Dodge, J. Paradiso, N. Gershenfeld, and D. Allport. Electric field sensing for graphical interfaces. IEEE Comput. Graph. Appl., 18(3):54-60, 1998.

[9] R. Wimmer, P. Holleis, M. Kranz, and A. Schmidt. Thracker - using capacitive sensing for gesture recognition. icdcsw, $0: 64,2006$

[10] T. G. Zimmerman, J. R. Smith, J. A. Paradiso, D. Allport, and N. Gershenfeld. Applying electric field sensing to human-computer interfaces. In $\mathrm{CHI}$ '95: Proceedings of the SIGCHI conference on Human factors in computing systems, pages 280-287, New York, NY, USA, 1995. ACM Press/Addison-Wesley Publishing Co. 